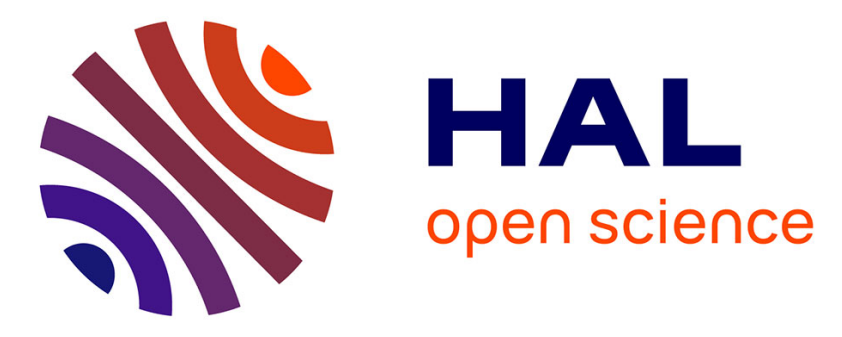

\title{
Spin current generation by ultrafast laser pulses in ferromagnetic nickel films
}

\author{
Jerome Hurst, Paul-Antoine Hervieux, Giovanni Manfredi
}

\section{To cite this version:}

Jerome Hurst, Paul-Antoine Hervieux, Giovanni Manfredi. Spin current generation by ultrafast laser pulses in ferromagnetic nickel films. Physical Review B: Condensed Matter and Materials Physics (1998-2015), 2018, 97 (1), 10.1103/PhysRevB.97.014424 . hal-02335713

\section{HAL Id: hal-02335713 \\ https://hal.science/hal-02335713}

Submitted on 28 Oct 2019

HAL is a multi-disciplinary open access archive for the deposit and dissemination of scientific research documents, whether they are published or not. The documents may come from teaching and research institutions in France or abroad, or from public or private research centers.
L'archive ouverte pluridisciplinaire HAL, est destinée au dépôt et à la diffusion de documents scientifiques de niveau recherche, publiés ou non, émanant des établissements d'enseignement et de recherche français ou étrangers, des laboratoires publics ou privés. 


\title{
Spin current generation by ultrafast laser pulses in ferromagnetic nickel films
}

\author{
Jérôme Hurst, Paul-Antoine Hervieux, and Giovanni Manfredi* \\ Université de Strasbourg, CNRS, Institut de Physique et Chimie des Matériaux de Strasbourg, UMR 7504, F-67000 Strasbourg, France
}

(Received 31 May 2017; revised manuscript received 4 December 2017; published 22 January 2018)

\begin{abstract}
A semiclassical phase-space model is used to study the ultrafast charge and spin dynamics in thin ferromagnetic films. Both itinerant and localized magnetism are taken into account. It is shown that an oscillating spin current can be generated in the film via the application of a femtosecond laser pulse in the visible range.
\end{abstract}

DOI: 10.1103/PhysRevB.97.014424

\section{INTRODUCTION}

Ultrafast magnetism has become a topical research area since the pioneering work of Beaurepaire et al. [1], who first observed the demagnetization of thin nickel films irradiated with femtosecond laser pulses. In spite of intense investigations (see the recent reviews [2,3]) the precise nature of such ultrafast demagnetization is still elusive. Suggested mechanisms include Elliott-Yafet electron-phonon spin-flip scattering [4,5], ultrafast magnon generation [6], and superdiffusive spin transport [7-9]. A recent theoretical approach based on time-dependent density functional theory (TD-DFT), which can potentially describe the exact charge and spin dynamics, has highlighted the role of the spin-orbit coupling in the demagnetization process $[10,11]$.

Understanding and controlling the magnetic properties of nano-objects on the subpicosecond time scale is an outstanding challenge of modern ultrafast science [12]. Spin currents have been recently shown to play a key role in the demagnetization process $[13,14]$. Here we show that an ultrafast spin current can be generated in a ferromagnetic nickel film via a femtosecond laser pulse of modest amplitude, for which the electric component of the pulse plays a preponderant role. In spite of this, a significant spin current can still be launched within the film and possibly observed through its emitted magnetic dipole radiation.

The results are obtained with a novel method $[15,16]$ based on the semiclassical evolution of a spin-dependent distribution function in the phase space, which models the dynamics of the conduction electrons and the associated itinerant magnetism, coupled to a Landau-Lifschitz equation for the fixed ion magnetism. The electron orbital motion is treated classically, whereas the spin is a fully quantum variable. All coupling constants are deduced from experimental results or first-principles considerations. This framework is intrinsically different from recent theoretical investigations of spin current generation [17], which employ a thermodynamic approach that assumes thermal equilibrium. In contrast, our approach is capable of dealing with the highly nonequilibrium scenarios that are expected to occur at such ultrafast time scales.

\footnotetext{
*giovanni.manfredi@ipcms.unistra.fr
}

\section{MODEL}

We consider a thin film of nanometric thickness $L$ much smaller than its transverse dimensions. In this configuration, it is appropriate to use a one-dimensional (1D) model, where only the longitudinal coordinate $x$, normal to the film surfaces, plays a role [Fig. 1(a)]. For the charge distribution of the ions, we use a jellium model with continuous ion density: $n_{i}(x)=n_{0}\{1+\exp [(|x|-L / 2) / \sigma]\}^{-1}$, where $\sigma$ is a diffuseness parameter $(\sigma \approx 1 \AA), n_{0}=\left(4 \pi r_{s} / 3\right)^{-1}$ is the ion density of the bulk metal, and $r_{s}$ is the Wigner-Seitz radius (for nickel, $r_{s}=2.6 a_{0}$, where $a_{0}$ is the Bohr radius).

Overall, our model is based on the distinction between itinerant magnetism (carried by the conduction electrons) and localized magnetism (carried by the fixed ions). The electronic structure of nickel is $\mathrm{Ni}=[\mathrm{Ar}] 3 d^{8} 4 s^{2}$. The $4 s$ electrons are assumed to be at the origin of the itinerant magnetism, whereas the $3 d$ electrons are localized around their nuclei to form ionic spins that are responsible for the localized magnetism [18]. The total magnetic moment of nickel $\boldsymbol{M}^{\text {tot }}=\boldsymbol{M}^{i}+\boldsymbol{M}^{e}$ is equal to $0.606 \mu_{B}$ per atom [19]. The magnetic moment of the localized ions is equal to $\boldsymbol{M}^{i}=0.54 \mu_{B}$ per atom at zero temperature [20], hence the magnetic moment of the itinerant $4 s$ electrons is $\boldsymbol{M}^{e}=0.066 \mu_{B}$ per atom. In the following we will refer to the itinerant $4 s$ electrons simply as the "electrons," and to the localized $3 d$ electrons and their nuclei as the "ions."

We take into account all the magnetic exchange interactions between the different spins. The exchange interaction between the ion spins is modeled using a Heisenberg exchange term [21] with coupling constant $J$ between nearest neighbors. The electron-ion exchange interaction is modeled by the Rundermann-Kittel-Kasuya-Yosida (RKKY) interaction [22], which is also a Heisenberg-type approach, with coupling constant $K$. The latter was originally developed in the Zener model [23] to describe the magnetism of transition metals, and was used in recent years to study the ultrafast magnetization dynamics in diluted magnetic semiconductors [24]. Finally, the electron-electron exchange interactions are modeled through appropriate exchange and correlation functionals $E_{\mathrm{xc}}\left[n_{e}\right]$. A sketch of the above-mentioned magnetic interactions is shown in Fig. 1(b).

The spin and charge dynamics of the itinerant electrons is modeled using a phase-space semiclassical approach that was developed in earlier works $[15,16]$. Several spin effects—such 

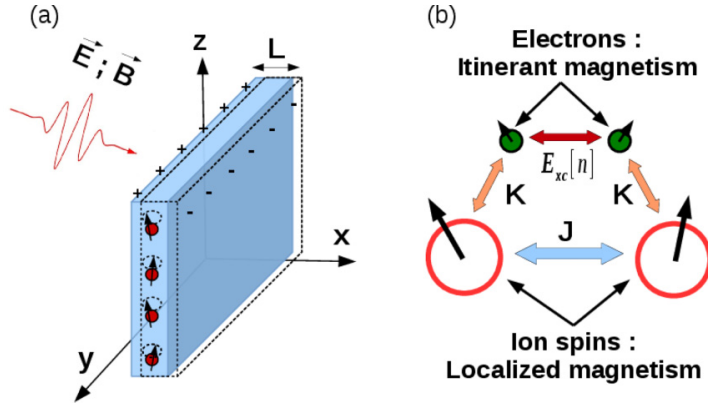

FIG. 1. (a) Geometry of a ferromagnetic thin film. (b) Schematic view of the various magnetic exchange interactions between the ion and electron spins. $K$ and $J$ are, respectively, the electron-ion and ion-ion coupling constants.

as the Zeeman term, the spin-orbit coupling, and higher-order relativistic corrections - can in principle be incorporated in this model. Here we shall only keep the Zeeman term, which allows us to reduce the phase space to $2 \mathrm{D}(x, v)$, and is sufficient to account for the spin current generation described later in this work. The electrons are described by a set of four phasespace distribution functions $f_{0}(x, v)$ and $f_{j}(x, v)(j=\{x, y, z\})$ which evolve according to the spin-Vlasov equations:

$$
\begin{gathered}
\frac{\partial f_{0}}{\partial t}+v \frac{\partial f_{0}}{\partial x}-\frac{1}{m} \frac{\partial V}{\partial x} \frac{\partial f_{0}}{\partial v}-\sum_{j} \frac{\mu_{B}}{m} \frac{\partial B_{j}}{\partial x} \frac{\partial f_{j}}{\partial v}=0 \\
\frac{\partial f_{j}}{\partial t}+v \frac{\partial f_{j}}{\partial x}-\frac{1}{m} \frac{\partial V}{\partial x} \frac{\partial f_{j}}{\partial v}-\frac{\mu_{B}}{m} \frac{\partial B_{j}}{\partial x} \frac{\partial f_{0}}{\partial v}-\frac{e}{m}[\boldsymbol{B} \times \boldsymbol{f}]_{j}=0 .
\end{gathered}
$$

The above equations can be seen as the semiclassical limit of a fully quantum model where the electron dynamics is represented by a $2 \times 2$ matrix Wigner function. In this approach, the orbital motion is treated classically (i.e., the electrons follow the classical trajectories), whereas the spin is a fully quantum variable $[15,16]$.

The distribution function $f_{0}$ represents the total electron density in the phase space, whereas $f_{j}$ is related to the spin polarization in the direction $j$. Indeed, integrating $f_{0}$ and $f_{j}$ over the velocity space gives, respectively, the electron density $n_{e}=\int f_{0} d v$ and the magnetization density $\boldsymbol{M}^{e}=$ $-\frac{\mu_{B}}{n_{0}} \int \boldsymbol{f} d v$.

The electric potential $V$ and the magnetic field $\boldsymbol{B}$ appearing in Eqs. (1) and (2) can be written as the sum of different contributions: $V=-e V_{H}+V_{\mathrm{xc}}+V_{\mathrm{ext}}$ and $\boldsymbol{B}=\boldsymbol{B}_{\mathrm{sd}}+\boldsymbol{B}_{\mathrm{xc}}+\boldsymbol{B}_{\mathrm{ext}}$. $V_{H}$ is the mean-field Hartree potential, solution to Poisson's equation:

$$
\epsilon_{0} \partial_{x}^{2} V_{H}=e\left(n_{e}-2 n_{i}\right)
$$

$\boldsymbol{B}_{\mathrm{sd}}=-K n_{i} \boldsymbol{S}^{i} / 2 \mu_{B}$ is the local magnetic field due the electron-ion exchange interactions, $V_{\mathrm{xc}}$ and $\boldsymbol{B}_{\mathrm{xc}}$ represent exchange-correlation effects in the local spin density approximation [25], while $V_{\text {ext }}$ and $\boldsymbol{B}_{\text {ext }}$ denote the external electromagnetic fields (laser pulse).

The ions are fixed, so that there is no charge dynamics associated with them. The ion spin dynamics is reduced to the precession of the spin vector $S^{i}(x, t)$, modeled by a continuous
Landau-Lifschitz (LL) equation [26]:

$$
\frac{\partial \boldsymbol{S}^{i}}{\partial t}=\frac{a^{2} J}{\hbar} \boldsymbol{S}^{i} \times \frac{\partial^{2} \boldsymbol{S}^{i}}{\partial x^{2}}-\gamma \boldsymbol{S}^{i} \times \boldsymbol{B}_{\mathrm{eff}},
$$

where $\gamma=g \mu_{B} / \hbar$ is the gyromagnetic ratio, $g$ is the Landé factor, $a=2 r_{s}$ is the interatomic distance, and $\boldsymbol{B}_{\text {eff }}=\boldsymbol{B}_{\text {ext }}+$ $K n_{0} \boldsymbol{M}^{e} / 2 g \mu_{B}^{2}$ is an effective magnetic field which incorporates the electron-ion spin exchange interaction through the coupling constant $K$. The ion magnetization density is then defined as $\boldsymbol{M}^{i}=-g \mu_{B} S^{i}$.

Equations (1)-(4) constitute a complete model to describe the charge and spin dynamics in ferromagnetic films. They are self-consistent, inasmuch as the electric field inside the film is the sum of two contributions: the laser field and the Hartree field created by all the electrons in response to the excitation.

In the forthcoming simulations, energies are normalized to the Fermi energy $E_{F}$, time to the inverse plasma frequency $\omega_{p}=\sqrt{e^{2} n_{0} / m \epsilon_{0}}$, velocities to the Fermi speed $v_{F}=$ $\sqrt{2 E_{F} / m}$, and lengths to $L_{F}=v_{F} / \omega_{p}$. For nickel one has $E_{F}=11.76 \mathrm{eV}, \omega_{p}^{-1}=0.058 \mathrm{fs}, v_{F}=1.12 \times 10^{6} \mathrm{~m} \mathrm{~s}^{-1}$, and $L_{F}=0.12 \mathrm{~nm}$

\section{FERROMAGNETIC GROUND STATE}

Before attacking the time-dependent problem, we need to compute the ground state of the system. For the electrons, the stationary solutions of Eqs. (1) and (2) can be written as

$$
\begin{aligned}
& f_{0}^{(0)}=\mathcal{F}_{\mathcal{D}}\left(H^{+}\right)+\mathcal{F}_{\mathcal{D}}\left(H^{-}\right), \quad f_{x}^{(0)}=0, \\
& f_{z}^{(0)}=\mathcal{F}_{\mathcal{D}}\left(H^{+}\right)-\mathcal{F}_{\mathcal{D}}\left(H^{-}\right), \quad f_{y}^{(0)}=0,
\end{aligned}
$$

where $\mathcal{F}_{\mathcal{D}}$ is the $1 \mathrm{D}$ Fermi-Dirac (FD) distribution

$$
\mathcal{F}_{\mathcal{D}}(H)=\frac{2 \pi k_{B} T}{m}\left(\frac{m}{2 \pi \hbar}\right)^{3} \ln \left\{1+\exp \left[(\mu-H) / k_{B} T\right]\right\},
$$

$H^{ \pm}=\frac{m}{2} v^{2}+V \pm \mu_{B} B_{z}$ and $\mu$ is the chemical potential. Equations (5) and (6) represent solutions for which the electron spins are polarized in the direction $z$ parallel to the film surfaces.

For the ions, the stationary solution of the LL equation (4) is given by the thermal average:

$$
\left\langle S_{z}^{i}\right\rangle=S \mathcal{B}_{S}\left[\frac{S}{k_{B} T}\left(2 J\left\langle S_{z}^{i}\right\rangle-\frac{K n_{0}}{2 \mu_{B}} M_{z}^{e}\right)\right],
$$

where $S=0.54 / g$ is the ion spin value at zero temperature and $\mathcal{B}_{S}$ is the $S$ th Brillouin function.

Equations (5)-(7) are formal solutions because they are expressed in terms of unknown quantities $\left(V_{H}, B_{\mathrm{sd}}, M^{e}\right)$, which depend themselves on the distribution functions. To find actual solutions, we employ an iterative procedure starting from an initial guess. This procedure depends on the two exchange constants $K$ and $J$. To find $K$, we set the temperature of the system to a low value (compared to the Curie temperature $T_{C}=$ $631 \mathrm{~K}$ ). Thus, for reasonable values of $J$, the ion magnetization is always maximal, i.e., $\left\langle S_{z}^{i}\right\rangle=S \widehat{e}_{z}$, and the magnetization of the itinerant electrons can be tuned with the parameter $K$ so that it matches the experimental value $\boldsymbol{M}^{\boldsymbol{e}}=0.066 \mu_{B}$ /atom Then we increase the temperature of the system and adapt 


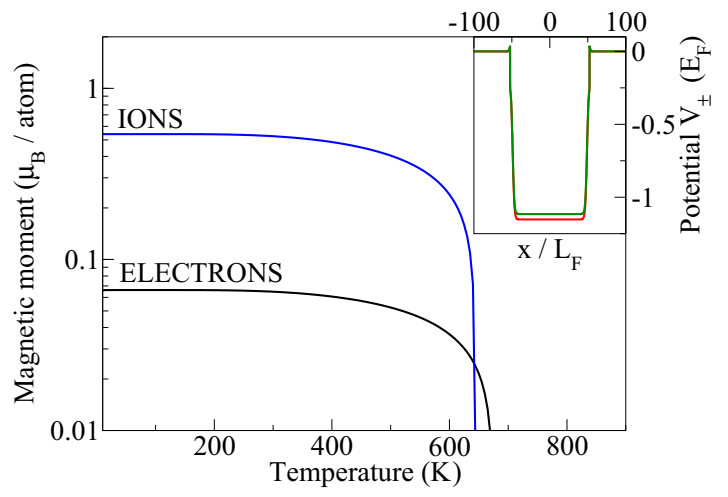

FIG. 2. Ground state of a nickel film with $L=100 L_{F}$ and $\sigma=L_{F}$. Main plot: Ion (blue curve) and electron (black curve) magnetizations as a function of the temperature. Inset: Effective confinement potentials $V_{ \pm}$for spin-up (red curve) and spin-down (green curve) electrons.

the parameter $J$ to obtain the correct Curie temperature. This procedure univocally determines the two exchange constants.

In Fig. 2 we plot the ground-state electron and ion magnetizations in a typical nickel film $\left(L=100 L_{F}, \sigma=L_{F}\right)$ as a function of the temperature. The coupling constants are $J=$ $0.022 \mathrm{eV}$ and $K=0.014 \mathrm{eV} \mathrm{nm}^{3}$; these values will be used throughout the rest of this work. The inset of Fig. 2 shows the spin-up $\left(V_{+}\right)$and spin-down $\left(V_{-}\right)$potentials: $V_{ \pm}=-e V_{H}+$ $V_{\mathrm{xc}} \pm \mu_{B}\left(B_{\mathrm{sd}}+B_{\mathrm{xc}}\right)$. The spin-up electrons are more strongly confined than the spin-down electrons, by approximately $0.05 \mathrm{eV}$. This difference originates from the Zeeman interaction and will be shown to be crucial in dephasing the dynamics of the spin-up and spin-down electrons, leading to the net spin current generation discussed in the next section.

\section{SPIN CURRENT GENERATION}

After computing the ferromagnetic ground state, we perturb it by shifting the distribution functions of a constant value $\Delta v$ [27]. This excitation corresponds to the application of an instantaneous electric field along the $x$ direction. A more realistic excitation (finite-duration laser pulse) will be analyzed later.

In Fig. 3 we show results for $\Delta v=0.05 v_{F}$. As it was observed in previous works [27,28], the electric dipole $\langle X\rangle_{e}=\int x f_{0} d x d v$ oscillates at the plasma frequency and, after few cycles, decays away because of Landau damping. What is more surprising is the creation of an oscillating magnetic dipole $\langle X\rangle_{m} \equiv \int x f_{z} d x d v$. This is unexpected, because we used a purely electrical excitation that perturbs both spin-up and spin-down electrons in the same way. An oscillating magnetic dipole is equivalent to an ac spin current, since $d\langle X\rangle_{m} / d t=\int v_{x} f_{z} d x d v=\int v_{x}\left(f_{\uparrow \uparrow}-\right.$ $\left.f_{\downarrow \downarrow}\right) d x d v=J_{\uparrow}-J_{\downarrow} \equiv J_{x z}^{S}$, where $J_{x z}^{S}$ is a spin current that propagates in the direction $x$ and is polarized along $z$ (in the general case $\boldsymbol{J}^{S}$ is a tensor, see [15]). This spin current is progressively created during the first few plasmon oscillations and then oscillates at a much lower frequency than the plasmon $\left(\approx 0.043 \omega_{p}\right)$. This is close to the ballistic frequency $\omega_{b} / 2 \pi=$ $v_{F} / 2 L$, i.e., the inverse of the time it takes to travel back and

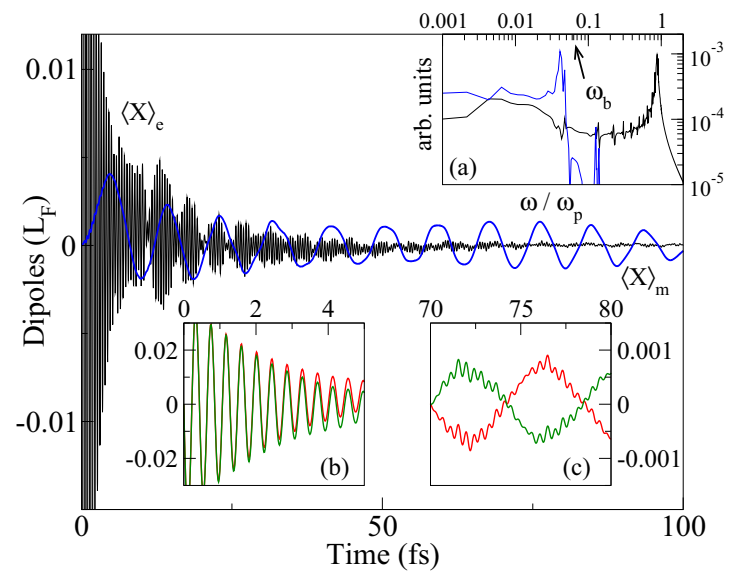

FIG. 3. Spin current generation in a nickel film with $L=50 L_{F}$ and $\sigma=L_{F}$, excited with a velocity shift $\Delta v=0.05 v_{F}$. Main plot: Time evolution of the electric (black curve) and magnetic (blue curve) dipoles. Inset (a) Fourier spectrum of the electric (black) and magnetic (blue) dipoles. Inset (b) Spin-up (red curve) and spin-down (green curve) dipoles in the early stages of the simulation (0-5 fs). Inset (c) Same as (b) at later times (70-80 fs).

forth in the film at the Fermi velocity, which for $L=50 L_{F}$ is equal to $0.063 \omega_{p}$ [29]. Simulations using thicker films confirmed that the frequency of the spin current oscillations scales ballistically as the inverse of $L$. Such spin oscillations persist for much longer times $(\approx 100 \mathrm{fs})$ after the electric dipole has damped away. Note that low-frequency ballistic oscillations were observed in earlier simulations on nonmagnetic films [27] and later confirmed by experimental measurements [30].

In order to explain the mechanism of spin current generation, we consider separately the spin-up and spin-down dipoles: $\langle X\rangle_{ \pm}=\frac{1}{2} \int x\left(f_{0} \pm f_{z}\right) d x d v$. In the ground state, the spin-up electrons are more strongly confined than the spindown electrons (see the inset of Fig. 2). This is due to the exchange interaction between the electron and the ion spins, which acts as a strong local magnetic field. After the initial electric excitation, both spin components are accelerated in the $x$ direction. The spin-down electrons, which are less deeply confined, will arrive at the film surface earlier than the spin-up electrons. At the surface, they experience a strong electric field that accelerates them back inside the film. Soon afterwards, the spin-up electrons will also reach the same surface, but the electric field they feel is slightly different from the one felt by the spin-down electrons, precisely because some of the latter are already present near the surface. Because of self-consistent electron-electron repulsion, the spin-up and spin-down dipoles will gradually start dephasing. The early stages of this dephasing can be seen in Fig. 3(b). This effect is cumulative and is thus amplified after each plasmon cycle until the two populations oscillate in antiphase [Fig. 3(c)], which corresponds to a magnetic dipole oscillation since $\langle X\rangle_{m}=\langle X\rangle_{+}-\langle X\rangle_{-}$. Overall, the effect corresponds to a transfer of momentum between the two spin populations, not directly via collisions but indirectly via the self-consistent Hartree field. It occurs near the surfaces because that is where the self-consistent field is largest, due to the strong density gradients. 

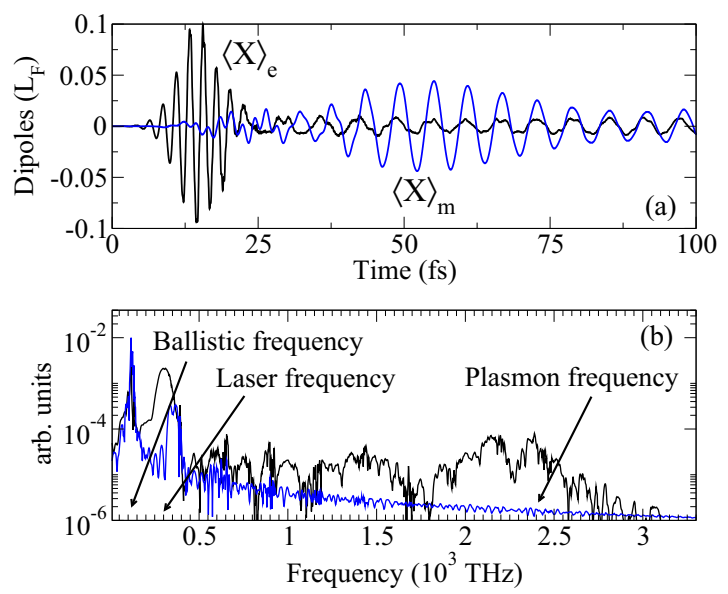

FIG. 4. Laser pulse excitation of a nickel film with $L=50 L_{F}$ and $\sigma=L_{F}$ (the laser specifications are given in the main text). (a) Time evolution of the electric (black curve) and magnetic (blue curve) dipoles. (b) Fourier spectrum of the dipoles computed using the full data $0<t<300$ fs.

In summary, three key ingredients were needed to create an oscillating magnetic dipole at the ballistic frequency with a purely electric excitation: (i) A magnetic ground state for which the spin-up and spin-down electrons are differently confined; (ii) a finite system with metal-vacuum interfaces near which the spin-up and spin-down dipoles are progressively dephased; and (iii) a self-consistent electric field which mediates momentum transfers between the two populations at such interfaces. If we suppress any one of the above ingredients, no spin current is generated.

\section{FEMTOSECOND LASER PULSE EXCITATION}

We now consider a more realistic scenario where the sample is excited by an ultrashort laser pulse. The pulse must be focused at an oblique angle of incidence with respect to the film surface, in order for the electric field to possess a longitudinal component $[31,32]$. Thus the electric field can be modeled as follows: $\boldsymbol{E}(x)=E_{0} \exp \left[-\left(t-t_{0}\right)^{2} / 2 \Delta t^{2}\right] \cos \left(\omega_{l} t\right) \widehat{\boldsymbol{e}}_{x}$, where $E_{0}$ is the amplitude of the field, $\Delta t$ and $\omega_{l}$ are, respectively, the duration and central frequency of the pulse, and $t_{0}$ is the time when the pulse reaches its maximal amplitude. The field is constant in space as its wavelength is much longer than the thickness of the film. Furthermore, the penetration depth of an electric field in nickel is about $10 \mathrm{~nm}$ in the visible and near-infrared range $[33,34]$. This is larger than the thickness of the films considered here $(6 \mathrm{~nm})$.

We excite the system with a laser pulse in the visible domain, with wavelength $\lambda_{l}=2 \pi c / \omega_{l}=800 \mathrm{~nm}$, duration
$2 \Delta t=14 \mathrm{fs}, t_{0}=15 \mathrm{fs}$, and amplitude $E_{0}=10^{10} \mathrm{~V} / \mathrm{m}$ (intensity $I \approx 13 \mathrm{TW} / \mathrm{cm}^{2}$, fluence $\approx 80 \mathrm{~mJ} / \mathrm{cm}^{2}$ ). In the frequency domain, this type of pulse covers a relatively narrow spectrum in the range $175-575 \mathrm{THz}$, near the ballistic frequency $\omega_{b} / 2 \pi=173 \mathrm{THz}$ (for a nickel film of thickness $L=50 L_{F}=$ $6 \mathrm{~nm}$ ) but far from the plasmon frequency $\omega_{p} / 2 \pi=2710 \mathrm{THz}$.

In Fig. 4(a) we show the time evolution of the electric and the magnetic dipoles during the first $100 \mathrm{fs}$. During the action of the laser pulse $(t<25 \mathrm{fs})$, the electric dipole unsurprisingly oscillates at the laser frequency. At the same time, a magnetic dipole is created. After the laser pulse, both the electric and the magnetic dipoles carry on oscillating at the ballistic frequency, signaling that the ballistic mode has been excited and persists even without the external drive.

These three oscillation modes-laser, ballistic, and plasmon-are seen as peaks in the frequency spectrum [Fig. 4(b)], with the strongest contribution coming from the ballistic mode. The plasmon mode is clearly, albeit weakly, excited for the electric dipole but is absent in the magnetic dipole spectrum, as expected. Its peak is significantly broader than the ballistic peaks, indicating that the plasmon is heavily Landau damped (see also Fig. 3) [35]. The plasmon mode could not be excited directly by the laser pulse, because it lies outside the laser spectrum, and must be due to the presence of nonlinear couplings.

\section{CONCLUSION}

We presented a realistic application of a semiclassical phase-space model to study ultrafast spin and charge transport in ferromagnetic thin films. The model combines both itinerant and localized magnetism and their interplay through various magnetic exchange mechanisms. Simulations of the interaction of a femtosecond laser pulse with a nickel film revealed the generation of spin currents propagating ballistically through the film. These currents appear as a result of the dephasing of the spin-up and spin-down itinerant electrons at the film surfaces. Their production is driven by nonequilibrium electrons, which is intrinsically different from the familiar Seebeck effect [37,38], i.e., the generation of a spin voltage by a temperature gradient. Our findings bear some similarities with recent experiments $[39,40]$ where spin currents were observed in multilayer structures due to the difference in transmittance between the spin-up and spin-down electrons at the $\mathrm{Au} / \mathrm{Fe}$ interface ("nonthermal Seebeck effect").

\section{ACKNOWLEDGMENT}

We acknowledge partial financial support from the Agence Nationale de la Recherche, Project No. ANR-11-LABX-0058NIE.
[1] E. Beaurepaire, J.-C. Merle, A. Daunois, and J.-Y. Bigot, Phys. Rev. Lett. 76, 4250 (1996).

[2] J.-Y. Bigot and M. Vomir, Ann. Phys. (Berlin) 525, 2 (2013).

[3] J. Walowski and M. Münzenberg, J. Appl. Phys. 120, 140901 (2016)
[4] K. Carva, M. Battiato, and P. M. Oppeneer, Phys. Rev. Lett. 107, 207201 (2011).

[5] B. Koopmans, G. Malinowski, F. D. Longa, D. Steiauf, M. Fähnle, T. Roth, M. Cinchetti, and M. Aeschlimann, Nat. Mater. 9, 259 (2010). 
[6] A. B. Schmidt, M. Pickel, M. Donath, P. Buczek, A. Ernst, V. P. Zhukov, P. M. Echenique, L. M. Sandratskii, E. V. Chulkov, and M. Weinelt, Phys. Rev. Lett. 105, 197401 (2010).

[7] M. Battiato, K. Carva, and P. M. Oppeneer, Phys. Rev. Lett. 105, 027203 (2010).

[8] J. Schellekens, W. Verhoeven, T. N. Vader, and B. Koopmans, Appl. Phys. Lett. 102, 252408 (2013).

[9] E. Turgut et al., Phys. Rev. Lett. 110, 197201 (2013).

[10] K. Krieger, J. K. Dewhurst, P. Elliott, S. Sharma, and E. K. U. Gross, J. Chem. Theory Comput. 11, 4870 (2015).

[11] K. Krieger, P. Elliott, T. Müller, N. Singh, J. K. Dewhurst, E. K. U. Gross, and S. Sharma, J. Phys.: Condens. Matter 29, 224001 (2017).

[12] T. J. Huisman, R. V. Mikhaylovskiy, J. D. Costa, F. Freimuth, E. Paz, J. Ventura, P. P. Freitas, S. Blügel, Y. Mokrousov, Th. Rasing, and A. V. Kimel, Nat. Nanotechnol. 11, 455 (2016).

[13] A. J. Schellekens, K. C. Kuiper, R. R. J. C. de Wit, and B. Koopmans, Nat. Commun. 5, 4333 (2014).

[14] G.-M. Choi, B.-C. Min, K.-J. Lee, and D. G. Cahill, Nat. Commun. 5, 4334 (2014).

[15] J. Hurst, O. Morandi, G. Manfredi, and P.-A. Hervieux, Eur. Phys. J. D 68, 176 (2014).

[16] J. Hurst, P.-A. Hervieux, and G. Manfredi, Philos. Trans. R. Soc. London Sect. A 375, 20160199 (2017).

[17] A. Fognini, T. U. Michlmayr, A. Vaterlaus, and Y. Acremann, J. Phys.: Condens. Matter 29, 214002 (2017).

[18] J. Stöhr, Magnetism From Fundamentals to Nanoscale Dynamics (Springer, Heidelberg, 2006).

[19] R. F. L. Evans, W. J. Fan, P. Chureemart, T. Ostler, M. O. Ellis, and R. W. Chantrell, J. Phys.: Condens. Matter 26, 103202 (2014).

[20] C. Kittel and P. McEuen, Introduction to Solid State Physics (Wiley, New York, 1996).

[21] W. Nolting and A. Ramakanth, Quantum Theory of Magnetism (Springer, Heidelberg, 2008).

[22] M. A. Ruderman and C. Kittel, Phys. Rev. 96, 99 (1954).

[23] C. Zener, Phys. Rev. 81, 440 (1951).
[24] O. Morandi, P.-A. Hervieux, and G. Manfredi, New J. Phys. 11, 73010 (2009).

[25] J. P. Perdew and A. Zunger, Phys. Rev. B 23, 5048 (1981).

[26] M. Lakshmanan, Philos. Trans. R. Soc. London Ser. A 369, 1280 (2011).

[27] G. Manfredi and P.-A. Hervieux, Phys. Rev. B 72, 155421 (2005).

[28] R. Jasiak, G. Manfredi, P.-A. Hervieux, and M. Haefele, New J. Phys. 11, 063042 (2009).

[29] The observed ballistic frequency is slightly smaller than the theoretical expression, because some electrons travel more slowly than $v_{F}$ and the effective thickness of the film is slightly larger than $L$, due to the soft edges [27].

[30] X. Liu, R. Stock, and W. Rudolph, Phys. Rev. B 72, 195431 (2005).

[31] M. Anderegg, Phys. Rev. Lett. 27, 1565 (1971).

[32] S. D. Brorson, J. G. Fujimoto, and E. P. Ippen, Phys. Rev. Lett. 59, 1962 (1987).

[33] V. Temnov, Nat. Photon. 6, 728 (2012).

[34] A. A. Grunin, A. G. Zhdanov, A. A. Ezhov, E. A. Ganshina, and A. A. Fedyanin, Appl. Phys. Lett. 97, 261908 (2010).

[35] The slight redshift with respect to the nominal plasma frequency, already observed in [27], is due to the fact that the computational box is finite, which - together with the 1D geometry and Dirichlet boundary conditions on $V_{H}$-allows normal modes with a frequency smaller than $\omega_{p}$ [36].

[36] F. C. Hoh, Phys. Rev. 133, A1016 (1964).

[37] H. Adachi, K.-I. Uchida, E. Saitoh, and S. Maekawa, Rep. Prog. Phys. 76, 036501 (2013).

[38] J. Kimling and D. G. Cahill, Phys. Rev. B 95, 014402 (2017).

[39] A. Alekhin, I. Razdolski, N. Ilin, J. P. Meyburg, D. Diesing, V. Roddatis, I. Rungger, M. Stamenova, S. Sanvito, U. Bovensiepen, and A. Melnikov, Phys. Rev. Lett. 119, 017202 (2017).

[40] I. Razdolski, A. Alekhin, N. Ilin, J. P. Meyburg, V. Roddatis, D. Diesing, U. Bovensiepen, and A. Melnikov, Nat Commun. 8, 15007 (2017). 